\title{
Circular RNAs as diagnostic tool for renal transplant patients with acute rejection
}

\author{
Joseph B. Moore $\mathrm{IV}^{1,2}$, Michael L. Merchant ${ }^{2,3}$, Shizuka Uchida ${ }^{1,2,4}$ \\ ${ }^{1}$ Diabetes and Obesity Center, ${ }^{2}$ The Christina Lee Brown Envirome Institute, Department of Medicine, ${ }^{3}$ Division of Nephrology and Hypertension, \\ Department of Medicine, ${ }^{4}$ Cardiovascular Innovation Institute, University of Louisville, Louisville, KY, USA \\ Correspondence to: Shizuka Uchida. Associate Professor of Medicine, Cardiovascular Innovation Institute, University of Louisville, KY 40202, USA. \\ Email: heart.lncrna@gmail.com; shizuka.uchida@louisville.edu. \\ Provenance: This is an invited article commissioned by the Guest Section Editor Dr. Ying Zhao (Department of Laboratory Medicine, the First \\ Affiliated Hospital, Zhejiang University School of Medicine, Hangzhou, China). \\ Comment on: Kölling M, Haddad G, Wegmann U, et al. Circular RNAs in Urine of Kidney Transplant Patients with Acute T Cell-Mediated Allograft \\ Rejection. Clin Chem 2019;65:1287-94.
}

Submitted Oct 17, 2018. Accepted for publication Oct 25, 2019.

doi: 10.21037/atm.2019.11.08

View this article at: http://dx.doi.org/10.21037/atm.2019.11.08

The kidney is a multifunctional organ that filters waste products from the blood in our body in the form of urine. In addition, kidneys also function to regulate blood pressure, balance electrolytes, and secrete erythropoietin to support erythrocyte (red blood cells) production. Severe loss of kidney function or kidney failure results in the accumulation of fluid and metabolic waste products-if left untreated, can lead to seizures, coma, or even death. Currently, the gold standard treatment for end-stage kidney disease patients is kidney transplantation (1). Unfortunately, as of 2016, the numbers of kidneys available for kidney transplantation are less than $17 \%$ of what are needed $(2,3)$. Hence significant effort had gone into preventing transplant rejection or transplant failure due to reoccurrence of the primary disease or the development of graft interstitial fibrosis and glomerulosclerosis (4). In effort to avoid such complication and support graft survival in recipients, patients must take immunosuppressive medications. When to start these medications depends on the severity of acute kidney rejection, which is classically measured by kidney allograft biopsy $(5,6)$. However, renal biopsy is invasive and carries potential associated risks to patient health. Noninvasive methods are preferred, which is a focus of intensive research in recent years (7-14).

Alternative splicing ("splicing") is a post-transcriptional process in which exons of nascent precursor messenger RNA (pre-mRNA) transcripts are included or excluded to form the mature mRNA (15). In humans, more than $90 \%$ of multi-exonic genes undergo splicing $(16,17)$. Due to its important contribution in diversifying protein isoforms from one gene, dysregulation in splicing (called "aberrant splicing") results in various human diseases, including renal diseases (18-21). Recent studies show that some spliced out exons and introns are not degraded in the nucleus, but an upstream $3^{\prime}$ splicing site (ss) can join with a downstream $5^{\prime}$ ss in a reversed order (called "backsplicing") to give rise to circular RNAs (circRNAs) (22-24). Since their discovery in early 1990s, studies have shown that circRNAs are quite stable and predominantly localize to the cytoplasmic compartment of cells $(25,26)$. One interesting feature of circRNAs is that they are more stable than that of noncircular RNAs (e.g., mRNA); this is due to the fact that circRNAs, which lack free 5' and $3^{\prime}$ ends, are not susceptible to RNA exonuclease-mediated degradation. Because of their stability, circRNAs accumulate in the blood (27-29), saliva (30), and urine (31), which have made them attractive candidates for biomarker discovery. Due to the less invasive (i.e., collection of blood) and non-invasive (i.e., saliva and urine) procedures necessary for their collection from patients, as compared to surgically procured/needle biopsy samples, circRNAs stand as an easily accessible diagnostic biomarker to potentially distinguish/identify various types of human diseases.

In a recent study by Kölling et al. (32), authors investigated 


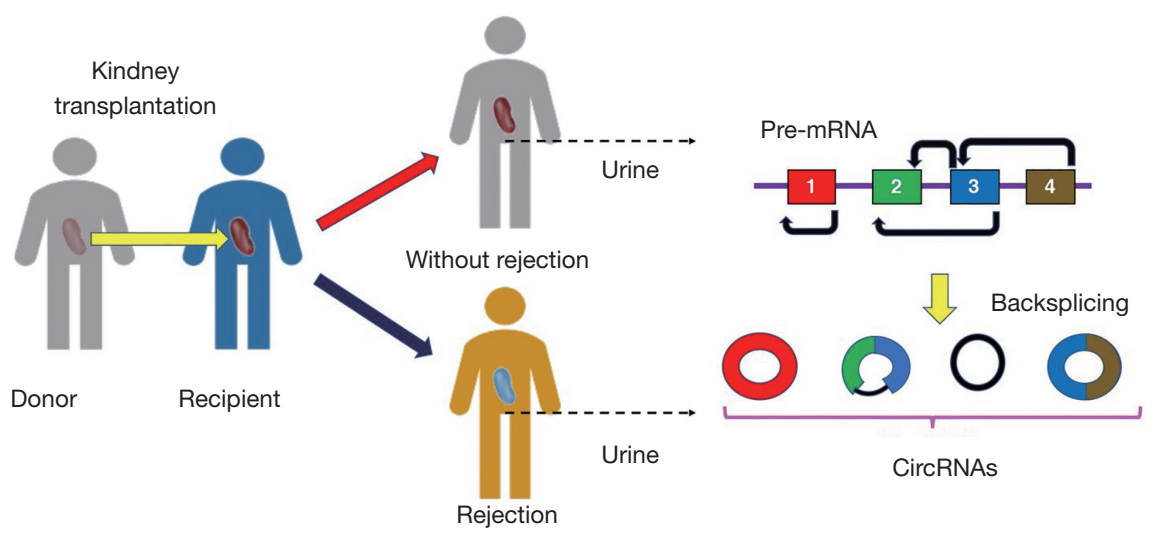

Figure 1 Detection of circRNAs from urine. Because circRNAs are more stable than linear mRNAs, Kölling et al. (32) proposed the usage of circRNAs in urine to distinguish kidney transplant patients with acute $\mathrm{T}$ cell-mediated allograft rejection from those without rejection.

the potential of using circRNAs in urine to distinguish kidney transplant patients with acute $\mathrm{T}$ cell-mediated allograft rejection from those without rejection (Figure 1). By utilizing human circRNA microarray (targeting 13,617 circRNAs), the authors detected 5,199 circRNAs in urine. Of these, 363 circRNAs were identified to be differentially expressed. Among these differentially expressed circRNAs, the authors chose two circRNAs, bsa_circ_0071475 and $b s a_{-}$ circ_0001334, for further analysis.

According to circBase (33), the database for circRNAs, bsa_circ_0071475 is located on chr 4:183,245,098-183,268,082 (GRCh37/hg19) and spans exons 1 and 2 of teneurin transmembrane protein 3 (TENM3) gene, which encodes for a transmembrane protein to regulate eye (34) and neuronal development (35). This circRNA was originally identified in Salzman et al. (36) and Rybak-Wolf et al. (37) to be expressed in cell lines (e.g., Hela, human ES cells) and various regions of brain (e.g., cerebellum, frontal cortex), respectively. Similarly, bsa_circ_0001334 is located on chr 3:127,337,917-127,341,124 (GRCh37/hg19) and spans exons 13 to 16 of minichromosome maintenance complex component 2 (MCM2) gene, which is involved in the initiation of genome replication (38). This circRNA was previously reported to be detected in CD19+ leukocytes (39). Since it is known that the expression pattern and function of circRNA may correlate with its parental gene (40), it is interesting to note that previous studies reported MCM2 can be used as prognostic proliferative marker in renal cell carcinoma (41) and Wilms tumor (42), suggesting that the selected circRNA, bsa_circ_0001334, can be originated from kidneys. Indeed, further validation experiments by Kölling et al. (32) show that the concentrations of bsa_circ_0001334 (but not bsa_circ_0071475) were significantly increased in patients with acute $\mathrm{T}$ cell-mediated rejection compared with stable transplant controls without signs of rejection. To further confirm the specificity of this differential expression, the authors also provide an evidence that the concentrations of this circRNA did not differ in kidney transplant patients with urinary tract infection. Based on these expression analyses, the authors propose bsa_circ_0001334 as a biomarker of acute renal allograft rejection, which can be detected in urine of patients to offer a non-invasive method.

As noted by the authors, it is a single-center cohort study. Thus, more rigorous, multi-center studies are needed to further confirm the validity of bsa_circ_0001334 as a biomarker. Given that circRNAs can be detected by RT-PCR assay, the primer pair can be designed at the backsplicing site to specifically target the circRNA but not its parental gene. Thus, the multi-center studies can be easily conducted using urine. Furthermore, the molecular mechanism of urinary circRNA release is not provided in this study, which is harder to investigate as the origin of circRNAs is extremely difficult to detect unless the parental gene is cell-type specifically expressed (more so than tissue specificity). However, as noted above, previous studies indicate the upregulation of MCM2, the parental gene of bsa_circ_0001334, in renal tumors $(41,42)$. Thus, it is plausible that aberrant splicing of MCM2 gene caused by deterioration in transplanted kidney may produce bsa_circ_0001334. However, a closer investigation about splicing variants of MCM2 gene is needed as there are nine MCM2 transcripts annotated in the Ensembl database (Ensembl Gene ID: ENSG00000073111), including five protein-coding, two nonsense mediated decay, one retained 
intron, and two lncRNAs. One lncRNA, MCM2-203 (Ensembl Transcript ID: ENST00000468659.1), spans between exons 12 and 16 of the longest isoform of MCM2 gene, MCM2-201 (Transcript ID: ENST00000265056.12), which corresponds to the majority of region covered by bsa_circ_0001334. As with any other IncRNA and circRNA research, rapid amplification of cDNA ends (RACE) and Northern blotting experiments must be performed to understand the whole transcriptomic length of bsa_circ_0001334 to delineate its distinction from another IncRNA encoded by the parental gene, MCM2.

In the last part of the Results section, the authors provide the in silico screening data of possible binding of miRNAs to bsa_circ_0001334. However, no direct, biological evidence (e.g., binding assay between circRNA and miRNAs) has not been provided, which raises a question about hsa_circ_0001334 functioning as miRNA sponges. Indeed, a comprehensive bioinformatics analysis (43) and our biological validation experiments (44) indicate that circRNAs acting as miRNA sponges are rare, while binding to RNA-binding proteins (RBPs) is more frequent (45-47) as some RBPs [e.g., Muscleblind (48), Quaking (49)] are involved in the biogenesis of circRNAs. Although it is outside of the scope of the current study, further detailed studies, including the characterization of bsa_circ_0001334 for its exact transcript length and biogenesis as well as gain/ loss-of-function experiments, are needed to understand the biology of circRNA in general.

In conclusion, the study by Kölling et al. (32) provides a nice addition to a repertoire of circRNAs detected in urine, which could serve as biomarkers for various diseases and their progressions, including acute renal allograft rejection investigated in this study.

\section{Acknowledgments}

Funding: This study was supported in part by National Institutes of Health Grant (P01 HL078825 to M.L.M.; R01-HL141081 to J.B.M.; P20 GM113226 to M.L.M.; P30 GM127607 to S.U.), V.V. Cooke Foundation (Kentucky, U.S.A.), and the startup funding from the Mansbach Family, the Gheens Foundation, and other generous supporters at the University of Louisville (to S.U.).

\section{Footnote}

Conflicts of Interest: The authors have no conflicts of interest to declare.
Ethical Statement: The authors are accountable for all aspects of the work in ensuring that questions related to the accuracy or integrity of any part of the work are appropriately investigated and resolved.

\section{References}

1. Mansell H, Rosaasen N, West-Thielke P, et al. Randomised controlled trial of a video intervention and behaviour contract to improve medication adherence after renal transplantation: the VECTOR study protocol. BMJ Open 2019;9:e025495.

2. Available online: https://www.kidney.org/news/newsroom/ factsheets/Organ-Donation-and-Transplantation-Stats

3. Available online: https://optn.transplant.hrsa.gov/

4. Yang C, Qi R, Yang B. Pathogenesis of Chronic Allograft Dysfunction Progress to Renal Fibrosis. Adv Exp Med Biol 2019;1165:101-16.

5. Singh N, Samant H, Hawxby A, et al. Biomarkers of rejection in kidney transplantation. Curr Opin Organ Transplant 2019;24:103-10.

6. Herath S, Erlich J, Au AYM, et al. Advances in Detection of Kidney Transplant Injury. Mol Diagn Ther 2019;23:333-51.

7. Yang JYC, Sarwal RD, Fervenza FC, et al. Noninvasive Urinary Monitoring of Progression in IgA Nephropathy. Int J Mol Sci 2019;20. doi: 10.3390/ijms20184463.

8. Van Loon E, Gazut S, Yazdani S, et al. Development and validation of a peripheral blood mRNA assay for the assessment of antibody-mediated kidney allograft rejection: A multicentre, prospective study. EBioMedicine 2019;46:463-72.

9. Christakoudi S, Runglall M, Mobillo P, et al. Development of a multivariable gene-expression signature targeting T-cell-mediated rejection in peripheral blood of kidney transplant recipients validated in cross-sectional and longitudinal samples. EBioMedicine 2019;41:571-83.

10. Banas M, Neumann S, Eiglsperger J, et al. Identification of a urine metabolite constellation characteristic for kidney allograft rejection. Metabolomics 2018;14:116.

11. Altug Y, Liang N, Ram R, et al. Analytical Validation of a Single-Nucleotide Polymorphism-Based DonorDerived Cell-Free DNA Assay for Detecting Rejection in Kidney Transplant Patients. Transplantation 2019. [Epub ahead of print].

12. Seo JW, Moon H, Kim SY, et al. Both absolute and relative quantification of urinary $\mathrm{mRNA}$ are useful for non-invasive diagnosis of acute kidney allograft rejection. 
PLoS One 2017;12:e0180045.

13. Hanssen O, Erpicum P, Lovinfosse P, et al. Non-invasive approaches in the diagnosis of acute rejection in kidney transplant recipients. Part I. In vivo imaging methods. Clin Kidney J 2017;10:97-105.

14. Hollis E, Shehata M, Abou El-Ghar M, et al. Statistical analysis of ADCs and clinical biomarkers in detecting acute renal transplant rejection. Br J Radiol 2017;90:20170125.

15. Black DL. Mechanisms of alternative pre-messenger RNA splicing. Annu Rev Biochem 2003;72:291-336.

16. Pan Q, Shai O, Lee LJ, et al. Deep surveying of alternative splicing complexity in the human transcriptome by highthroughput sequencing. Nat Genet 2008;40:1413-5.

17. Wang ET, Sandberg R, Luo S, et al. Alternative isoform regulation in human tissue transcriptomes. Nature 2008;456:470-6.

18. Horita S, Simsek E, Simsek T, et al. SLC4A4 compound heterozygous mutations in exon-intron boundary regions presenting with severe proximal renal tubular acidosis and extrarenal symptoms coexisting with Turner's syndrome: a case report. BMC Med Genet 2018;19:103.

19. Low KJ, Ansari M, Abou Jamra R, et al. PUF60 variants cause a syndrome of ID, short stature, microcephaly, coloboma, craniofacial, cardiac, renal and spinal features. Eur J Hum Genet 2017;25:552-9.

20. Reynolds DM, Hayashi T, Cai Y, et al. Aberrant splicing in the PKD2 gene as a cause of polycystic kidney disease. J Am Soc Nephrol 1999;10:2342-51.

21. Peral B, Gamble V, San Millan JL, et al. Splicing mutations of the polycystic kidney disease 1 (PKD1) gene induced by intronic deletion. Hum Mol Genet 1995;4:569-74.

22. Jeck WR, Sharpless NE. Detecting and characterizing circular RNAs. Nat Biotechnol 2014;32:453-61.

23. Jeck WR, Sorrentino JA, Wang K, et al. Circular RNAs are abundant, conserved, and associated with ALU repeats. Rna 2013;19:141-57.

24. Boeckel JN, Jae N, Heumuller AW, et al. Identification and Characterization of Hypoxia-Regulated Endothelial Circular RNA. Circ Res 2015;117:884-90.

25. Cocquerelle C, Mascrez B, Hetuin D, et al. Mis-splicing yields circular RNA molecules. Faseb j 1993;7:155-60.

26. Nigro JM, Cho KR, Fearon ER, et al. Scrambled exons. Cell 1991;64:607-13.

27. Zhuang ZG, Zhang JA, Luo HL, et al. The circular RNA of peripheral blood mononuclear cells: Hsa_circ_0005836 as a new diagnostic biomarker and therapeutic target of active pulmonary tuberculosis. Mol Immunol 2017;90:264-72.
28. Memczak S, Papavasileiou P, Peters O, et al. Identification and Characterization of Circular RNAs As a New Class of Putative Biomarkers in Human Blood. PLoS One 2015;10:e0141214.

29. Kolling M, Seeger H, Haddad G, et al. The Circular RNA ciRs-126 Predicts Survival in Critically Ill Patients With Acute Kidney Injury. Kidney Int Rep 2018;3:1144-52.

30. Zhao SY, Wang J, Ouyang SB, et al. Salivary Circular RNAs Hsa_Circ_0001874 and Hsa_Circ_0001971 as Novel Biomarkers for the Diagnosis of Oral Squamous Cell Carcinoma. Cell Physiol Biochem 2018;47:2511-21.

31. Vo JN, Cieslik M, Zhang Y, et al. The Landscape of Circular RNA in Cancer. Cell 2019;176:869-81.e13.

32. Kölling M, Haddad G, Wegmann U, et al. Circular RNAs in Urine of Kidney Transplant Patients with Acute T Cell-Mediated Allograft Rejection. Clin Chem 2019;65:1287-94.

33. Glazar P, Papavasileiou P, Rajewsky N. circBase: a database for circular RNAs. RNA 2014;20:1666-70.

34. Leamey CA, Merlin S, Lattouf P, et al. Ten_m3 regulates eye-specific patterning in the mammalian visual pathway and is required for binocular vision. PLoS Biol 2007;5:e241.

35. Tran H, Sawatari A, Leamey CA. The glycoprotein Ten-m3 mediates topography and patterning of thalamostriatal projections from the parafascicular nucleus in mice. Eur J Neurosci 2015;41:55-68.

36. Salzman J, Chen RE, Olsen MN, et al. Cell-type specific features of circular RNA expression. PLoS Genet 2013;9:e1003777.

37. Rybak-Wolf A, Stottmeister C, Glazar P, et al. Circular RNAs in the Mammalian Brain Are Highly Abundant, Conserved, and Dynamically Expressed. Mol Cell 2015;58:870-85.

38. Eide T, Tasken KA, Carlson C, et al. Protein kinase A-anchoring protein AKAP95 interacts with MCM2, a regulator of DNA replication. J Biol Chem 2003;278:26750-6.

39. Memczak S, Jens M, Elefsinioti A, et al. Circular RNAs are a large class of animal RNAs with regulatory potency. Nature 2013;495:333-8.

40. Meng X, Hu D, Zhang P, et al. CircFunBase: a database for functional circular RNAs. Database (Oxford) 2019;2019. doi: 10.1093/database/baz003.

41. Dudderidge TJ, Stoeber K, Loddo M, et al. Mcm2, Geminin, and KI67 define proliferative state and are prognostic markers in renal cell carcinoma. Clin Cancer Res 2005;11:2510-7. 
42. Taran K, Sitkiewicz A, Andrzejewska E, et al. Minichromosome maintenance 2 (MCM2) is a new prognostic proliferative marker in Wilms tumour. Pol J Pathol 2011;62:84-8.

43. Guo JU, Agarwal V, Guo H, et al. Expanded identification and characterization of mammalian circular RNAs. Genome Biol 2014;15:409.

44. Militello G, Weirick T, John D, et al. Screening and validation of $\operatorname{lncRNAs}$ and circRNAs as miRNA sponges. Brief Bioinform 2017;18:780-8.

45. Abdelmohsen K, Panda AC, Munk R, et al. Identification of HuR target circular RNAs uncovers suppression of PABPN1 translation by CircPABPN1. RNA Biol 2017;14:361-9.

Cite this article as: Moore JB 4th, Merchant ML, Uchida S. Circular RNAs as diagnostic tool for renal transplant patients with acute rejection. Ann Transl Med 2019;7(Suppl 8):S302. doi: 10.21037/atm.2019.11.08
46. Dudekula DB, Panda AC, Grammatikakis I, et al. CircInteractome: A web tool for exploring circular RNAs and their interacting proteins and microRNAs. RNA Biol 2016;13:34-42.

47. Schneider T, Hung LH, Schreiner S, et al. CircRNAprotein complexes: IMP3 protein component defines subfamily of circRNPs. Sci Rep 2016;6:31313.

48. Ashwal-Fluss R, Meyer M, Pamudurti NR, et al. circRNA biogenesis competes with pre-mRNA splicing. Mol Cell 2014;56:55-66.

49. Conn SJ, Pillman KA, Toubia J, et al. The RNA binding protein quaking regulates formation of circRNAs. Cell 2015;160:1125-34. 